Supporting Information for:

\title{
Chimeric Peptides Quickly Modify the Surface of Personalized 3D Printing Titanium Implant to Promote Osseointegration
}

Zhezhe Zhao ${ }^{\sharp, a} \quad$ Shiqing Ma $a^{\#, a}$ Chenxuan $W u^{a} \quad$ Xuewen Li ${ }^{a} \quad$ Xinying $M a^{a}$

Han $\mathrm{Hu}^{a} \quad$ Jie Wu ${ }^{* a} \quad$ Yonglan Wang*a $\quad$ Zihao Liu**aa

a. School and Hospital of Stomatology, Tianjin Medical University, Tianjin 300070, People's Republic of China

Corresponding Author

*E-mail: liuzihao@tmu.edu.cn (Zihao Liu)

*E-mail: wujiedoctor@tmu.edu.cn (Jie Wu)

* E-mail: tmuperiodontology@163.com (Yonglan Wang) 


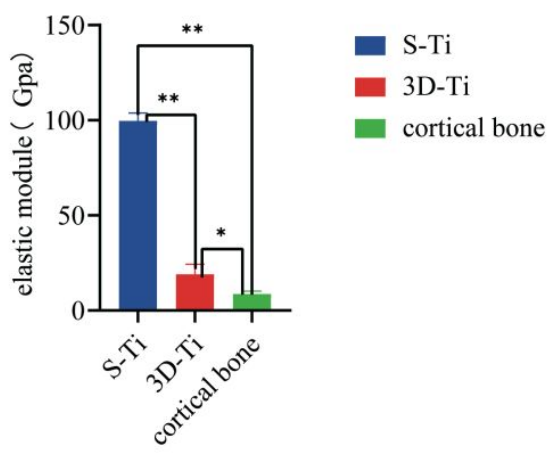

Figure S1. The elastic modulus of S-Ti and 3D-Ti. 